\section{INCREASED INJURY RISK IN YOUTH ATHLETICS WHEN GROWTH RATES ARE HIGH AND SKELETAL MATURATION IS LOW}

1,2Eirik Halvorsen Wik, ${ }^{1}$ Daniel Martínez-Silván, ${ }^{1}$ Abdulaziz Farooq, 3,4Marco Cardinale, ${ }^{1}$ Amanda Johnson, ${ }^{1,2}$ Roald Bahr. 'Aspetar Orthopaedic and Sports Medicine Hospital, Doha, Qatar; ${ }^{2}$ Norwegian School of Sport Sciences, Oslo, Norway; ${ }^{3}$ Aspire Academy, Doha, Qatar; ${ }^{4}$ University College London, London, UK

\subsection{6/bjsports-2021-IOC.3}

Background Studies addressing risk factors for injuries in youth athletics are scarce and although growth and maturation represent potential risk factors for adolescent athletes, the available literature is inconclusive.

Objective The aim of this study was to examine if growth rate, maturity status and maturity tempo are associated with injury risk in adolescent athletics.

Design Anthropometric, skeletal maturity and injury data collected prospectively over four seasons were included.

Setting The data collection was part of the ongoing monitoring of athletes at the Aspire Academy in Qatar.

Patients (or Participants) Participants were student-athletes, not yet specialized to event groups. Of the 129 athlete-seasons eligible for inclusion, 117 athlete-seasons (74 athletes) were included in the final sample.

Interventions (or Assessment of Risk Factors) Anthropometric measures were taken at the start and end of each season, while skeletal maturity was assessed at the start of each season using hand radiographs.

Main Outcome Measurements Time-loss injuries were recorded by medical staff and associations were assessed using generalized estimating equations.

Results Growth rate for stature was associated with greater risk of bone (Incidence rate ratio (IRR): 1.5 per SD above the mean, 95\% CI: 1.1 to 1.9$)$ and growth plate injuries $(2.1,1.5$ to 3.1). Growth rate for leg length was associated with greater risk of overall $(1.3,1.0$ to 1.7$)$, bone $(1.4,1.0$ to 1.9$)$ and growth plate injuries $(2.1,1.4$ to 3.0$)$. Athletes with greater maturity status $(0.6$ per skeletal age year, 0.5 to $0.9 ; 0.8$ per percent of mature height; 0.7 to 1.0 ) were less prone to growth plate injuries. Annual change in skeletal age was associated with an increased risk of bone injuries (1.5 per SD above the mean; 1.0 to 2.3 ).

Conclusions The results of this study suggest that rapid growth in stature and leg length, skeletal maturity status and maturity tempo represent risk factors for certain injury types in adolescent athletics. Regular monitoring therefore seems warranted.

\section{IS INFERIOR DUAL-TASK PERFORMANCE A RISK FACTOR FOR INJURY IN YOUTH SOCCER? A PROSPECTIVE STUDY}

Evi Wezenbeek, Dries Pieters, Joke Schuermans, Tine Willems, Erik Witvrouw. Ghent University, Ghent, Belgium

\subsection{6/bjsports-2021-IOC.4}

Background The effects of dual task (DT) performance on injury incidence in sports have barely been researched. Athletes often encounter situations where cognitive and motor tasks must be performed simultaneously, with interference between tasks. An inferior DT performance could be associated with a less adequate (motor) response, increasing the injury risk.

Objective To examine whether inferior DT performance could be identified as a risk factor for injury in elite youth soccer.

Prospective cohort study.

Setting Soccer-specific DT performance was evaluated by means of the Loughborough Soccer Passing Test (LSPT), followed by a prospective monitoring period (injury and sports exposure registry).

Participants 130 male youth soccer players, aged $12.8 \pm 1.7$ years

Assessment of Risk Factors Cox regression analyses, corrected for age, were performed to identify DT performance as a potential risk factor for traumatic injuries.

Main Outcome Measurements (1) LSPT test and LSPT test with additional cognitive task (LSPT + ) scores, as well as the (2) Relative Dual Task Interference (RDTI).

Results $32 \%$ of players sustained an injury, resulting in a mean time loss of $16.12 \pm 22.87$ days. Overall injury incidence was 2.86 per 1000 exposure hours. The performance in both single and dual task conditions improved with age $(\mathrm{p}<0.001)$. Meanwhile, the RDTI remained relatively constant across all age groups $(p=0.816)$. Survival analyses revealed no significant predictive effects of DT performance on injury occurrence. Age was significantly able to predict injury occurrence $(p=0.011)$, with older players demonstrating a higher risk.

Conclusions Inferior DT performance could not be associated with increased injury risk in youth soccer. Age was a significant risk factor for injury incidence in this sample but did not present any association with DT performance. DT performance capacity cannot be seen as a risk factor for injury in youth soccer.

\section{HOW DO FOOTBALL (SOCCER) INJURIES OCCUR? A SYSTEMATIC VIDEO ANALYSIS OF 345 MODERATE AND SEVERE MATCH INJURIES}

${ }^{1,2}$ Christian Klein, ${ }^{3}$ Patrick Luig, ${ }^{2}$ Thomas Henke, ${ }^{1}$ Hendrik Bloch, ${ }^{2}$ Petra Platen. ${ }^{1} V B G$, German Statutory Accident Insurance for the Administrative Sector, Department of Sports Injury Prevention, Hamburg, Germany; ${ }^{2}$ Ruhr-Universität Bochum, Faculty of Sports Science, Department of Sports Medicine and Sports Nutrition, Bochum, Germany; ${ }^{3}$ German Handball Federation, Dortmund, Germany

\subsection{6/bjsports-2021-IOC.5}

Background Although descriptions of injury inciting events are of key importance to understand the causes of any particular injury type, systematic analysis of inciting events remain rare.

Objective Identification and description of recurrent injury patterns in professional football.

Design Prospective video analysis of moderate and severe match injuries.

Setting Two highest divisions in German professional male football (Bundesliga, 2. Bundesliga).

Patients (or Participants) All players who played at least one competitive club match within the seasons 2014/15, 15/16 and $16 / 17$ were included $(n=1,449)$.

Interventions (or Assessment of Risk Factors) Systematic video analysis of all moderate and severe match injuries (time-loss $>$ 8 days) that were registered by clubs or physicians with the VBG (German statutory accident insurance for professional athletes) as part of the occupational accident reporting. 